$56-57$

\title{
Accuracy and reliability of MRI quantitative measurements to assess spinal cord compression in cervical spondylotic myelopathy: a prospective study
}

Authors Alina Karpova, Sorin Craciunas, Soo-Yong Chua, Doron Rabin, Sean Smith, Michael G Fehlings Institution Toronto Western Hospital, Spinal Surgery Program, University of Toronto, Canada

\section{ABSTRACT}

Study type: Reliability study

Introduction: Cervical spondylotic myelopathy (CSM) is the most common spinal cord disorder in persons more than 55 years old. Despite multiple neuroimaging approaches proposed to quantify the spinal cord compromise in CSM patients, magnetic resonance imaging (MRI) remains the procedure of choice by providing helpful information for clinical decision making, determining optimal subpopulations for treatment, and selecting the optimal treatment strategies. However, the validity, reliability, and accuracy of the MRI quantitative measurements have not yet been addressed.

Objective: To assess the intra- and inter-observer reliability of MRI quantitative measurements of the spinal cord compromise in CSM patients.

Methods: Seventeen CSM patients (13 male) of mean age 54.5 years old were selected from the AOSpine North America database. The patients had different combinations of stenotic levels (1-4 levels) and the clinical severity (range mJOA baseline: 8-18). Asymptomatic or previous surgically treated CSM, active infection, neoplastic disease, rheumatoid arthritis, ankylosing spondylitis, trauma, or concomitant lumbar stenosis were excluded. The patients underwent preoperative MRI using $1.5 \mathrm{~T}$ (15 patients) and 3T (two patients) scanner, including mid-sagittal T1-weighted, axial and mid-sagittal T2weighted series. MRI data were analyzed (Mango 2.0 software; Multi-Image Analysis GUI) by four blind raters in three different sessions. Four measurements were analysed: transverse area (TA)

This research was funded by AOSpine North America. The protocol was approved by the UHN Research Ethics Board 
(Figure 1), compression ratio (CR) (Figure 2), maximal canal compromise (MCC), and maximal spinal cord compression (MSCC) (Figure 3). The differences for each measurement were evaluated using mixed-effect ANOVA models (ratter, session, ratter $x$ session). The intra- and inter-rater reliability was evaluated with intraclass correlation coefficients (ICC) (Figure 4).

Results: The principal findings were: (i) for TA $\left(71.48 \pm 12.99 \mathrm{~mm}^{2}\right)$, the intra-rater agreement was 0.97 (95\% CI, range 0.94-0.99) and the inter-rater agreement was 0.76 (95\% CI, range 0.49-0.90); (ii) for CR $(0.35 \pm 0.04 \%), 0.94(95 \% \mathrm{CI}$, range $0.88-$ $0.98)$, and 0.79 (95\% CI, range $0.57-0.91)$ respectively; (iii) for MCC $(83.21 \pm 2.08 \%), 0.95$ (95\% CI, range $0.89-0.98)$, and 0.64 (95\% CI, range $0.28-0.85)$ respectively; and (iv) for MSCC (82.87 $\pm 1.52 \%), 0.93$ (95\% CI, range $0.86-0.97)$, and 0.84 (95\% CI, range $0.65-0.93)$ respectively.

Conclusions: Our data suggest that three out of four measurements (TA, CR and MSCC) have acceptable intraand interreliability coefficients (ICC > 0.75). However, for the maximal canal compromise measure, although the intrareliability was acceptable, the interrater reliability was not acceptable (0.64). Based on this study, we recommend that three MRI measures: transverse area, compression ratio and maximal spinal cord compression should be used in the imaging assessment of the spinal cord in CSM patients.

\section{REFERENCES:}

1. Okada Y, Ikata T, Yamada H, et al (1993) Magnetic resonance imaging study on the results of surgery for cervical compression myelopathy. Spine; 15;18(14):2024-2029.

2. Bednarik J, Kadanka Z, Dusek L, et al (2008) Presymptomatic spondylotic cervical myelopathy: an updated predictive model. Eur Spine J; 17(3):421-431.

3. Fehlings MG, Rao SC, Tator CH, et al (1999) The optimal radiologic method for assessing spinal canal compromise and cord compression in patients with cervical spinal cord injury. Part II: Results of a multicenter study. Spine;. 15;24(6):605613.

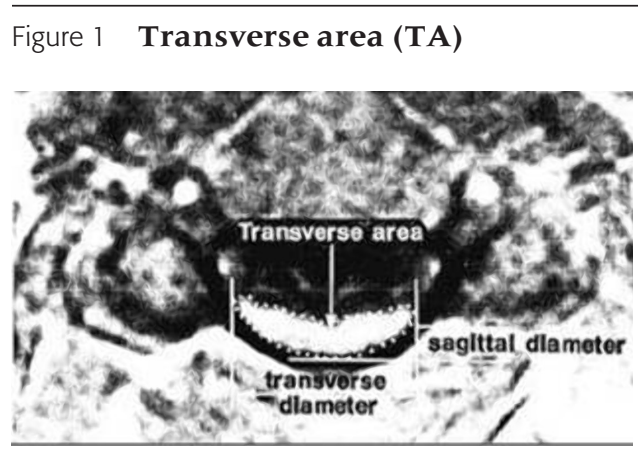

Figure 2 Compression ratio $(\mathbf{C R}=\mathbf{A P} / \mathbf{W})$

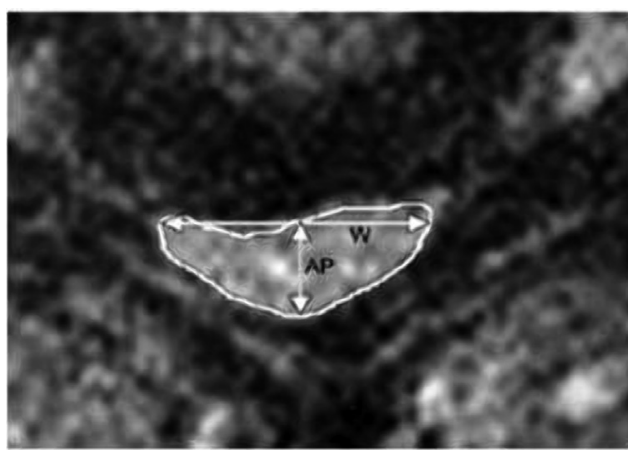

Figure 3 Maximal canal compromise (MCC), and maximal spinal cord compression (MSCC). $\operatorname{MCC}(\%)=1-[D x /(D a+D b) / 2] \times 100 \%$; $\operatorname{MSCC}(\%)=1-[d x /(d a+d b) / 2] \times 100 \%$
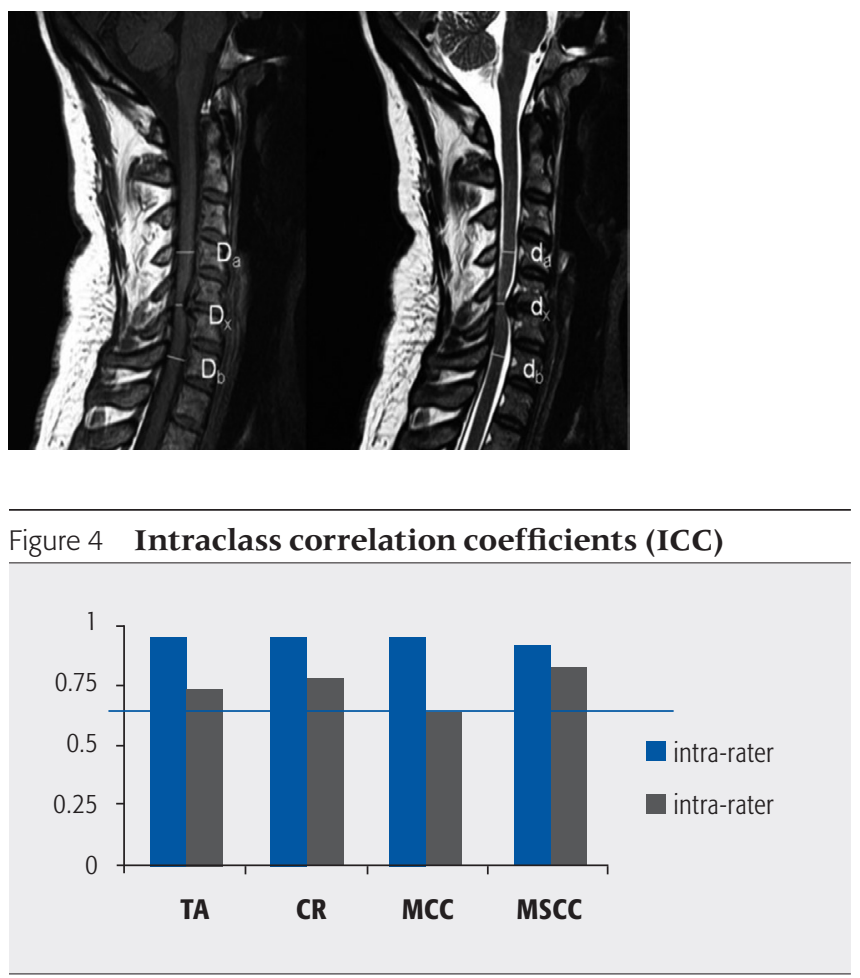